\title{
Muon capture on nuclei with $N>Z$, random phase approximation, and in-medium value of the axial-vector coupling constant
}

\author{
E. Kolbe \\ Physics Department, University of Basel, CH-4056 Basel, Switzerland \\ K. Langanke \\ Institute for Physics and Astronomy, University of Aarhus, DK-8000 Aarhus, Denmark \\ P. Vogel \\ Physics Department, California Institute of Technology, Pasadena, California 91125
}

(Received 6 June 2000; published 9 October 2000)

\begin{abstract}
We use the random phase approximation to describe the muon capture rate on ${ }^{44} \mathrm{Ca},{ }^{48} \mathrm{Ca},{ }^{56} \mathrm{Fe},{ }^{90} \mathrm{Zr}$, and ${ }^{208} \mathrm{~Pb}$. With ${ }^{40} \mathrm{Ca}$ as a test case, we show that the continuum random phase approximation (CRPA) and the standard RPA give essentially equivalent descriptions of the muon capture process. Using the standard RPA with the free nucleon weak form factors we reproduce the experimental total capture rates on these nuclei quite well. Thus, unlike the allowed $\beta$ decays, with very small momentum transfer and exclusively the $1^{+}$multipole, the muon capture rate, with momentum transfer of approximately muon mass magnitude, and a mixture of multipoles (dominated by $1^{-}$), does not require any quenching of the axial-vector coupling constant.

PACS number(s): 24.30.Cz, 23.40.Hc
\end{abstract}

The capture of a negative muon from the atomic $1 s$ orbit,

$$
\mu^{-}+(Z, N) \rightarrow \nu_{\mu}+(Z-1, N+1)^{*}
$$

is a semileptonic weak process which has been studied for a long time (see, e.g., the reviews by Walecka [1] or Mukhopadhyay [2] and the earlier references therein). The total capture rate has been measured for many nuclei [3]; in some cases the partial capture rates to specific states in the daughter nucleus have been determined as well.

The nuclear response in muon capture is governed by the momentum transfer which is of the order of the muon mass. The energy transferred to the nucleus is restricted from below by the mass difference of the initial and final nuclei, and from above by the muon mass. The phase space and the nuclear response favor lower nuclear excitation energies. There is an intimate relation between the inclusive muon capture rate and the cross section for the antineutrinoinduced charged-current reactions; both are governed by the same nuclear matrix elements and proceed from the same initial to the same final states.

Since the experimental data are quite precise, and the theoretical techniques of evaluating the nuclear response in the relevant regime are well developed, it is worthwhile to see to what extent the capture rates are theoretically understood. This is not only interesting per se, but should be viewed as a more general test of our ability to describe semileptonic weak charged-current reactions.

A step in this direction was undertaken by us several years ago in Ref. [4] where the continuum random phase approximation (CRPA) was used to describe muon capture on the $N=Z$ nuclei ${ }^{12} \mathrm{C},{ }^{16} \mathrm{O}$, and ${ }^{40} \mathrm{Ca}$. We showed that the method allows us to reproduce the experimental total capture rates on these nuclei to better than $10 \%$ using the free nucleon weak form factors and two different residual interactions. In particular, it was not necessary to apply the in- medium quenching of the axial vector coupling constant. This is contrary to various well-known indications that the axial-vector coupling constant $g_{A}$ in nuclear medium is reduced from its free nucleon value of $g_{A}=1.26$ to the value of $g_{A} \simeq 1$ when one analyzes the data on $\beta$ decay between lowlying states of the $(s d)$ shell nuclei $[5]$ and $(p f)$ shell nuclei [6]. In addition, the "missing Gamow-Teller strength", problem, as revealed in the interpretation of the forward-angle $(p, n)$ and $(n, p)$ charge-exchange reactions [7], is also often quoted as evidence for quenching of $g_{A}$. Note that the Gamow-Teller (GT) strength is concentrated in the giant GT resonance at excitation energies not very far from the energies involved in the muon capture, although this latter process is usually dominated by the transitions to the negative parity spin-dipole states. That is so in particular in the double-magic nuclei ${ }^{16} \mathrm{O}$ and ${ }^{40} \mathrm{Ca}$ where the GT strength is strongly suppressed, while in ${ }^{12} \mathrm{C}$ it is essentially exhausted by the transition to the analog of the $T=1$ state at 15.11 $\mathrm{MeV}$ in ${ }^{12} \mathrm{C}$, whose contribution to the muon capture rate has been subtracted in [4]. It is thus of interest to inquire whether a similar quenching applies in muon capture over a broader range of nuclei.

There is a consensus that the quenching of $g_{A}$ in $\beta$ decay [and $(p, n)$ reactions] is related to the neglect of configurations outside the usual $0 \hbar \omega$ shell model space and/or the effect of meson-exchange currents. Thus, generally, one expects that the quenching phenomenon might be multipolarity and momentum transfer dependent. Evaluation of the muon capture rate makes it possible to extend the quenching study to higher multipoles. Knowing whether, and to what extent, the quenching is needed to describe muon capture rates has practical application as well. For example, evaluation of the cross section for supernova neutrino detectors, or of the matrix elements for the neutrinoless double $\beta$ decay involves higher than $1^{+}$multipoles, and larger momentum transfers, 
than ordinary $\beta$ decay. Another application, already mentioned, involves the neutrino (and antineutrino) induced nuclear reactions, e.g. from the neutrinos associated with the pion and muon decays at rest.

In this paper we extend the previous calculation [4] to heavier nuclei, in particular nuclei with the neutron excess, i.e., with a nonvanishing value of the initial isospin. In [4] the continuum random phase approximation (CRPA) was used, a method shown to be successful for the description of the nuclear response to weak and electromagnetic probes [8]. The method combines the usual RPA treatment with the correct description of the continuum nucleon decay channel. For heavier $N>Z$ nuclei that method is computationally quite demanding. Moreover, it can describe only transitions to states in the daughter nucleus above the particle emission threshold. In order to evaluate the total capture rate one has to include also the transitions to bound states, which contribute relatively more, and are typically not experimentally separated in the heavier nuclei.

If one is interested in the total capture rate, the numerically simpler standard RPA is just as good. As an additional bonus, it avoids the distinction between the bound and unbound states. That the two methods, CRPA and standard RPA, are equivalent for the present purpose is demonstrated in Fig. 1 for the case of ${ }^{40} \mathrm{Ca}$. We show in the upper part the differential capture rate as a function of the excitation energy in the final nucleus. While the CRPA (for details see Ref. [8]) is characterized by a continuous curve, nonvanishing everywhere above the threshold, the standard RPA is characterized by the "picket fence," since there is a finite number of discrete final states. The similarity of both methods is even better seen in the lower part of Fig. 1 which shows the integrated rate up to a given excitation energy $E^{*}$. There appears to be a slight systematic shift of a few MeV (caused by the bound state contribution, presumably), but the final capture rates, and the typical excitation energies, are remarkably similar. Thus, we use the standard RPA for the evaluation of the muon capture in the selected $N>Z$ nuclei.

For the calculation in the present work we used the phenomenological Landau-Migdal force with parameters that has been shown to be applicable for a wide range of nuclei [9]. All single-particle states below the Fermi level were included and two oscillator shells above it were taken into account. Using again ${ }^{40} \mathrm{Ca}$ as a test case, we checked that adding or subtracting in the calculation few subshells above the Fermi level does not visibly change the muon capture rate. However, in ${ }^{208} \mathrm{~Pb}$ enlarging the single particle space leads to an increase of the capture rate by about $5 \%$. The free nucleon form factors were used to describe the weak nuclear current. In particular, the unquenched axial vector coupling constant $g_{A}(0)=1.26$ was used.

Muon capture also depends on the induced pseudoscalar hadronic weak current. At the free nucleon level the corresponding coupling constant is determined by the GoldbergerTreiman relation [10]

$$
F_{P}\left(q^{2}\right)=\frac{2 M_{p} g_{A}(0)}{m_{\pi}^{2}-q^{2}}
$$
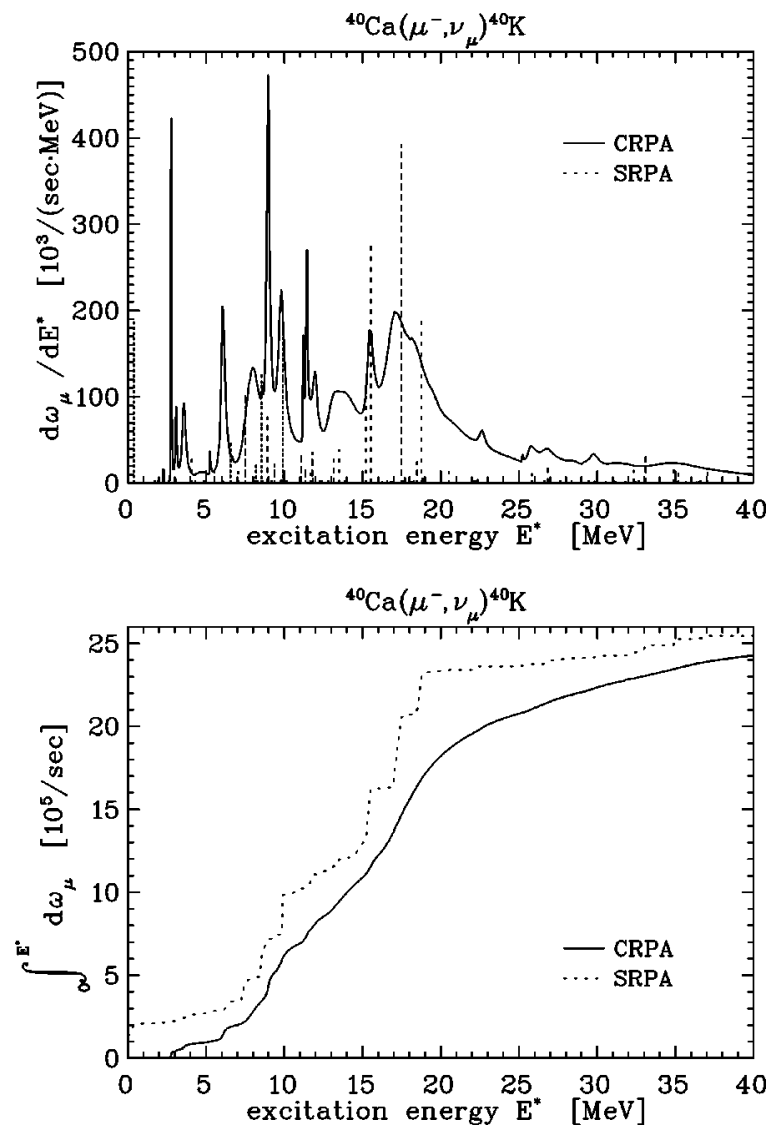

FIG. 1. The $\mu^{-}$capture rate as a function of the excitation energy in the final nucleus ${ }^{40} \mathrm{~K}$ (upper panel). The continuous curve is for the continuum random phase approximation (CRPA), while the dashed vertical bars are the results of the standard random phase approximation (SRPA). In the lower panel the integrated $\mu^{-}$capture rate, up to the excitation energy $E^{*}$, is shown.

where $m_{\pi}$ is the pion mass and $g_{A}(0)=1.26$. [In muon capture one often uses a dimensionless quantity $g_{P}$ $=m_{\mu} F_{P}\left(q^{2}\right)$ at the relevant momentum transfer $q^{2} \simeq$ $-0.9 m_{\mu}^{2}$, such that $g_{P} \simeq 8.4$ for free protons.] In nuclear medium $F_{P}$ can be renormalized again, and this renormalization does not necessarily obey the Goldberger-Treiman relation [11]. We have shown in our previous work that the total muon capture rates are not sensitive enough to the various choices of $F_{P}$ renormalization. Consequently, throughout this work we use the Goldberger-Treiman relation.

The calculated total capture rates are collected in Table I and compared with the data [3]. For comparison we also show in Table I the earlier results [4] for the $N=Z$ nuclei, evaluated with the same residual Landau-Migdal force. Among the nuclei in Table I, ${ }^{56} \mathrm{Fe}$ is the only one with a substantial contribution of the Gamow-Teller transitions, which are known to be quenched. Hence the $1^{+}$part of the capture rate was quenched by the empirical factor of 2.54 obtained by comparing the total experimental and calculated GT strength. This reduced $1^{+}$rate was then combined with the unquenched rate from the other multipoles in column 3 of Table I. Clearly, a better agreement with experiment for all considered nuclei is achieved when the full value of $g_{A}$ is 
TABLE I. $\mu^{-}$-capture rates calculated within the standard (SRPA) and continuum (CRPA) models in units of $10^{3} \mathrm{~s}$. The radius $r$ and diffuseness $d$ of the extended nuclear charge distribution were set to $(r, d)$ $=(1.07 \mathrm{fm}, 0.50 \mathrm{fm})$ for ${ }^{12} \mathrm{C}$ and $(r, d)=(1.07 \mathrm{fm}, 0.57 \mathrm{fm})$ for all other nuclei. The Landau-Migdal force (LM) is used throughout.

\begin{tabular}{lccccc}
\hline \hline Nucleus & Expt. [3] & $\begin{array}{c}\text { SRPA } \\
g_{A}(0)=1.26\end{array}$ & $\begin{array}{c}\text { SRPA } \\
g_{A}(0)=1.0\end{array}$ & $\begin{array}{c}\text { CRPA(LM) } \\
g_{A}(0)=1.26\end{array}$ & $\begin{array}{c}\text { CRPA(LM) } \\
g_{A}(0)=1.0\end{array}$ \\
\hline${ }^{12} \mathrm{C}$ & $32.8 \pm 0.8$ & & & $31.3^{\mathrm{c}}$ & $22.9^{\mathrm{c}}$ \\
${ }^{16} \mathrm{O}$ & $102.6 \pm 0.6$ & & & 103.2 & 75.8 \\
${ }^{40} \mathrm{Ca}$ & $2544 \pm 7^{\mathrm{a}}$ & 2547 & 1846 & 2489 & \\
${ }^{44} \mathrm{Ca}$ & $1793 \pm 40$ & 1722 & 1238 & & \\
${ }^{48} \mathrm{Ca}$ & $1164^{\mathrm{b}}$ & 1301 & 930 & \\
${ }^{56} \mathrm{Fe}$ & $4400 \pm 100$ & $4460^{\mathrm{c}}$ & $3430^{\mathrm{c}}$ & & \\
${ }^{90} \mathrm{Zr}$ & $9350 \pm 100$ & 10288 & 7400 & & \\
${ }^{208} \mathrm{~Pb}$ & $13450 \pm 180$ & 16057 & 11436 & & \\
\hline \hline
\end{tabular}

${ }^{\mathrm{a}}$ Corrected from the data for natural Ca.

${ }^{b}$ Extrapolated using the Primakoff formula fitted to ${ }^{40} \mathrm{Ca}$ and ${ }^{44} \mathrm{Ca}$.

${ }^{c}$ Calculated with partial occupation of the single particle subshells; see [17].

used. The quenched value leads to an obvious underestimate of the muon capture rate.

In Fig. 2 the fractional contributions of different multipoles are shown for ${ }^{16} \mathrm{O},{ }^{48} \mathrm{Ca},{ }^{90} \mathrm{Zr}$, and ${ }^{208} \mathrm{~Pb}$. For the closed shell nucleus ${ }^{16} \mathrm{O}$ the negative parity $1^{-}$and $2^{-}$, and to lesser extent $0^{-}$, multipoles dominate, as expected. In the intermediate mass nuclei, ${ }^{48} \mathrm{Ca}$ and ${ }^{90} \mathrm{Zr}$, the dominance of these multipolarities is less pronounced. Finally, in ${ }^{208} \mathrm{~Pb}$ the positive parity $1^{+}$and $2^{+}$multipoles give the largest contribution, since the negative parity proton hole-neutron particle states are blocked. Note, however, that these $1^{+}$and $2^{+}$ states correspond to the $2 \hbar \omega$ excitations involving different major shells, not the usual $0 \hbar \omega$ Gamow-Teller transitions.

The dominance of dipole transitions for the $\mu^{-}$capture process in the $p$ and $s, d$ shell nuclei is a well-known phe-

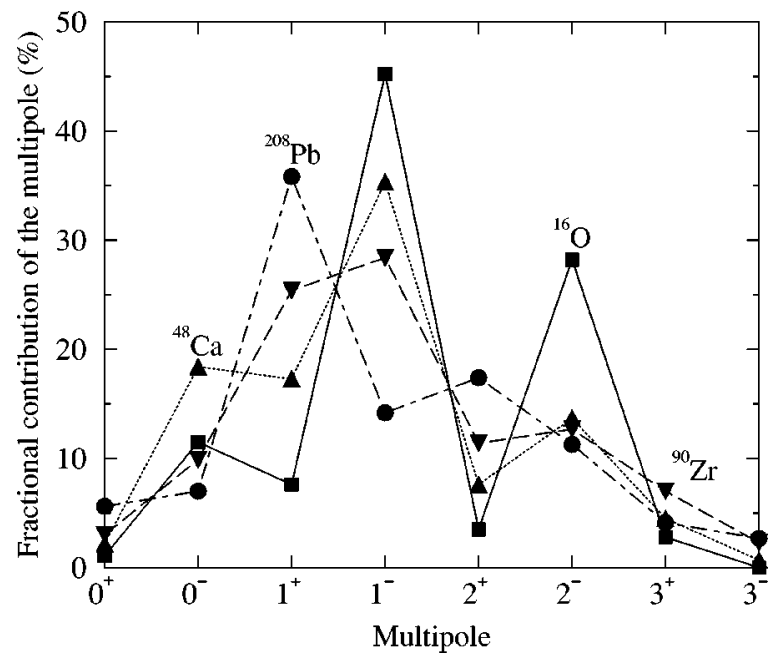

FIG. 2. Fractional contribution of different multipoles to the total capture rate for four of the considered nuclei. The entries with $I^{\pi}=0^{-}, 1^{-}, 2^{-}$correspond to the $1 \hbar \omega$ excitations, while the entries with $I^{\pi}=1^{+}, 2^{+}, 3^{+}$correspond to the $2 \hbar \omega$ excitations. The data points are connected by lines for better visibility and the nuclei are as indicated in the figure. nomenon. It can be exploited in the generalized GoldhaberTeller model [12], where all dipole and spin-dipole strength is concentrated in a single collective state. The GoldhaberTeller model has been applied to the muon capture already a long time ago [12]. Here we have repeated the calculation with one modification. Instead of using as the energy of the single collective state the energy $E_{D}$ of the giant dipole resonance in the initial nucleus, we placed the strength into a state at the excitation energy $E^{*}=E_{D}-E_{I A S}$ in the final nucleus, where $E_{I A S}$ is the energy of the isobar analog state with $T=1$ in the initial $N=Z$ nucleus. Such an assignment puts the strength close to the centroid of the excitation spectrum obtained in the RPA. The method is rather crude, since more detailed calculations clearly show that the spin-dipole strength is spread over a sizable energy interval. Nevertheless, the results displayed in Table II, obtained again with the full value of $g_{A}(0)=1.26$, support our conclusion that there is no quenching in these nuclei of the operators of the weak current that change parity.

First forbidden $\beta$ decays, in particular those with $\Delta I^{\pi}$ $=0^{-}$, have been often analyzed as a source of information on the enhancement caused by the meson exchange currents. This enhancement, whose origin is however well understood, is an example of the multipolarity dependence of the apparent in-medium effects. In the context of the work reported here it is worthwhile to quote the work of Warburton and Towner [13] who analyzed 18 first forbidden $\beta$ decays in

TABLE II. $\mu^{-}$-capture rates calculated within the GoldhaberTeller model in units of $10^{3} \mathrm{~s}$. The formulas of Ref. [12] are used. In column 2 the original parameters are employed. In column 3 the excitation energy $E^{*}$ is modified, as explained in the text.

\begin{tabular}{lccc}
\hline \hline Nucleus & Expt. [3] & Orig. values [12] & Modified $E^{*}$ \\
\hline${ }^{12} \mathrm{C}$ & $32.8 \pm 0.8$ & 29.7 & 35.1 \\
${ }^{16} \mathrm{O}$ & $102.6 \pm 0.6$ & 79.2 & 123 \\
${ }^{28} \mathrm{Si}$ & $871 \pm 2$ & 657 & 970 \\
${ }^{40} \mathrm{Ca}$ & $2544 \pm 7$ & 1490 & 2730 \\
\hline \hline
\end{tabular}


the lead region. They used a truncated shell model and found that the $\Delta I^{\pi}=1^{-}$transitions, which are unaffected by the meson exchange currents, do not require any quenching. In fact, their fit to an overall quenching factor for the first forbidden matrix elements results in $s q_{1}=0.98 \pm 0.05$, compatible with unity, i.e., with no quenching. That analysis, totally independent of our evaluation of the muon capture, again supports the conclusion about the apparent absence of an appreciable quenching of the parity-changing (i.e., first forbidden) weak current operators.

The unique second forbidden $\beta$ decays are governed by a single operator $r^{2}\left[Y_{2} \sigma\right]^{\lambda=3}$. For only a handful of them the partial decay rates are known. When analyzed [14,15], these transitions do not allow one to draw any definitive conclusion about the possible quenching of the corresponding strength. However, as pointed out above, $\mu^{-}$capture rate in ${ }^{90} \mathrm{Zr}$, and ${ }^{208} \mathrm{~Pb}$, is strongly affected by the $2 \hbar \omega$ transitions, whose operators are related to the second forbidden $\beta$ decays. Since the agreement between the experimental and calculated rates in these two nuclei, and in particular in ${ }^{208} \mathrm{~Pb}$, is not as good as in the other cases, we cannot make a strong statement regarding the quenching of the positive parity "second forbidden" multipoles based on the muon capture calculations.

In conclusion, the present analysis shows that the CRPA and SRPA methods are capable of describing the total $\mu^{-}$ capture rates quite well in a large range of nuclei. The dependence of the muon capture rate on the isospin, the socalled Primakoff rule [16], is also reasonably well reproduced. There is no indication of the necessity to apply any quenching to the operators responsible for the $\mu^{-}$process. Thus our findings can be used as guidance in the evaluation of other semileptonic weak processes involving higher multipoles, i.e., transitions involving other than the $0 \hbar \omega$ spin changing operators.

This work was supported in part by the Swiss National Science Foundation, the Danish Research Council, and by the U.S. Department of Energy, Contract No. DE-F60388ER-40397.
[1] J. D. Walecka, in Muon Physics II, edited by V. W. Hughes and C. S. Wu (Academic, New York, 1975), p. 113.

[2] N. C. Mukhopadhyay, Phys. Rep., Phys. Lett. 30C, 1 (1977).

[3] T. Suzuki, D. F. Measday, and J. P. Roalsvig, Phys. Rev. C 35, 2212 (1987).

[4] E. Kolbe, K. Langanke, and P. Vogel, Phys. Rev. C 50, 2576 (1994).

[5] B. H. Wildenthal, Prog. Part. Nucl. Phys. 11, 5 (1984).

[6] E. Caurier, A. Poves, and A. P. Zuker, Phys. Rev. Lett. 74, 1517 (1995).

[7] C. D. Goodman and S. B. Bloom, in Spin Excitations in $\mathrm{Nu}$ clei, edited by F. Petrovich et al. (Plenum, New York, 1983), p. 143; G. F. Bertsch and H. Esbensen, Rep. Prog. Phys. 50, 607 (1987); O. Häusser et al., Phys. Rev. C 43, 230 (1991).

[8] M. Buballa, S. Drozdz, S. Krewald, and J. Speth, Ann. Phys. (N.Y.) 208, 346 (1991); E. Kolbe, K. Langanke, S. Krewald, and F. K. Thielemann, Nucl. Phys. A540, 599 (1992).

[9] G. A. Rinker and J. Speth, Nucl. Phys. A306, 360 (1978); M. R. Plunket et al., Phys. Rev. C 56, 263 (1997).

[10] M. L. Goldberger and S. B. Treiman, Phys. Rev. 111, 354 (1958).

[11] J. Delorme and M. Ericson, Phys. Rev. C 49, R1763 (1994).

[12] T. W. Donnelly, J. Dubach, and W. C. Haxton, Nucl. Phys. A251, 353 (1975).

[13] E. K. Warburton and I. S. Towner, Phys. Rep. 243, 103 (1994).

[14] G. Martinez-Pinedo and P. Vogel, Phys. Rev. Lett. 81, 281 (1998).

[15] E. K. Warburton, Phys. Rev. C 45, 463 (1992).

[16] H. Primakoff, Rev. Mod. Phys. 31, 802 (1959).

[17] E. Kolbe, K. Langanke, and P. Vogel, Nucl. Phys. A652, 91 (1999). 